\title{
Lapping behavior as a function of the reinforcement schedule on another manipulandum'
}

William Wagman and Cynthia Seheuer ${ }^{2}$ SOUTHERN ILLINOIS UNIVERSITY

\begin{abstract}
Abstraet
Lapping behavior of rats was investigated as a function of the ratio reinforcement schedule on another manipulandum in an operant learning situation. The data indicated that this member of the operant chain was an increasing function of the size of the ratio, although the dipper behavior was experimentally independent, of the requirements of the schedule. Problem

This study was the first in a series in which attempts will be made to investigate more than one response measure in an operant learning situation. In 1962, Weissman presented data demonstrating that licking rates at a milk reinforcement dipper increased as the interval between reinforcements increased on a variable-interval reinforcement schedule. The present study was an attempt to investigate the lapping behavior of rats at a water reinforcement dipper as a function of the ratio reinforcement schedule on another manipulandum. The behaviors investigated were bar-press rates on fixed-ratio schedules and lapping behavior at the reinforcement dipper at different values on the ratio parameter. The study was designed to demonstrate that the schedule which the animal is subjected to on one manipulandum influences other members of the operant response chain.

\section{Method}

The subjects were six one hundred day old male laboratory rats.

The apparatus consisted of a standard Lehigh Valley operant conditioning apparatus with a $.06 \mathrm{cc}$ water reinforcement dipper. All contingencies were programmed by means of relay switching circuits. A print-out counter recorded the number of laps the animal made at each dipper presentation. Laps were measured by a standard Grayson-Stadler drinkometer which was connected to the dipper and grid bars of the box.

Animals were subjected to a 23-hr. water deprivation cycle throughout the experiment. Initially, the Ss were trained to bar press for water on a modified continuous reinforcement schedule (CRF) in which they had to lap at the dipper at each presentation in order to produce further reinforcement. Animals were run on CRF to a satiation criterion of no responding for a 10-min. period, However, if the criterion was reached in less than $1 \mathrm{hr}$., the Ss remained in the apparatus until the criterion was reached again. At stabilization on CRF, the distribution of laps per dipper presentation was recorded for a three-day period and averaged to provide mean lapping distribu-

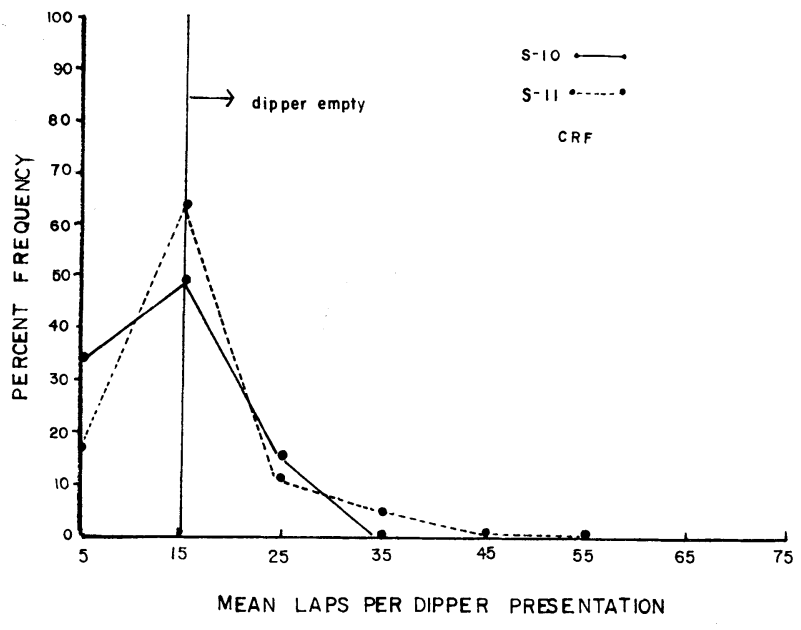

Fig. 1. Lapping distributions of two animals on CRF. The ordinate represents the percentage of reinforcements in which $\mathrm{S}$ lapped at the dipper a particular number of times during an experimental session. The absicca represents the mean number of laps made at each dipper presentation.

tions for each animal. After the lapping distributions were recorded on $\mathrm{CRF}$, the animals were subjected to fixed-ratio (FR) schedules of $10,20,30$, and 60 responses. Animals were run under each of these schedules until their behavior had stabilized, and their lapping distributions were recorded in the same manner as previously on CRF.

\section{Results and Discussion}

The data for Ss 10 and 11 on CRF indicates that the modal laps per dipper presentation is at 15 . Stellar \& Hill (1952) have data which indicate that a rat picks up from .004 to $.005 \mathrm{cc}$ of water at each lap. Thus, with a dipper size of $.06 \mathrm{cc}, 12$ to 15 laps are all that is necessary for the animal to pick up all of the reinforcement presented. Figure 1 demonstrates that animals, when subjected to CRF, do not consume all of the water in the dipper at each presentation. These animals left some reinforcement in the dipper approximately $40 \%$ of the time.

Figure 2 represents the lapping distributions for Ss $1,2,4$, and 6 on FR schedules of $10,20,30$, and 60 responses. The mode of the distributions for $\mathrm{S}-2$ does not change from a schedule of FR 10 to FR 20, nor from FR 20 to FR 30, although it does become more skewed with a greater proportion of laps now occurring at the higher intervals. At FR 60, however, 

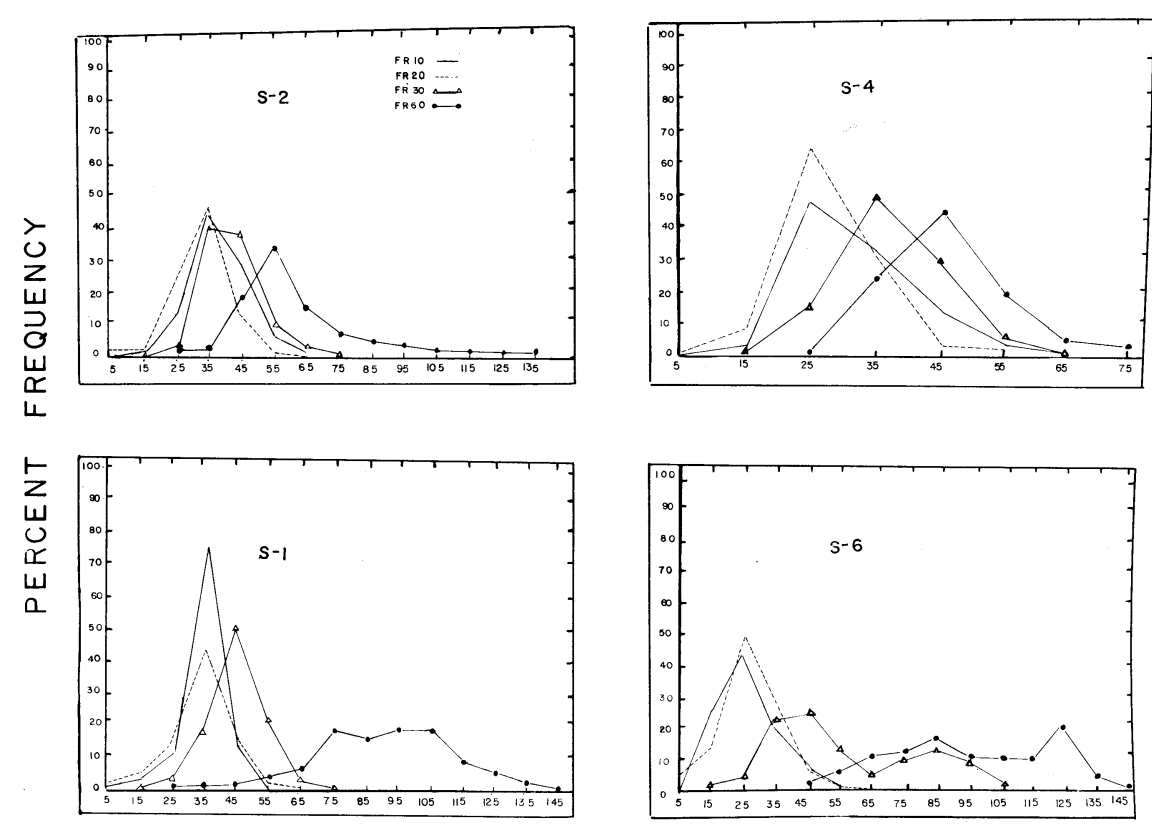

Fig. 2. Lapping distributions for Ss $1,2,4$, and 6 on FR schedules of $10,20,30$, and 60 responses.

\section{MEAN LAPS/DIPPER PRESENTATION}

the mode moves form 35 to 55 , a jump of 20 laps per dipper presentation.

The mode of the distributions for S-4 remains at 25 at FR values of 10 and 20 , but moves up to 35 on an FR 30 schedule and to 45 on an FR 60 schedule.

The mode of the distributions for $\mathrm{S}-1$ is at 25 at FR schedules of 10 and 20 and is nearly normal. This animal's kymograph records indicated evidence of ratio strain when subjected to an FR 30 schedule and an FR. 60 schedule as well. These distributions were not only flatter than under the other ratio schedules but showed evidence of bimodality with peaks at the higher lapping intervals of 45 and again at 85 (FR 30), and at 85 and 125 (FR 60).

The mode of the distributions for $\mathrm{S}-6$ on ratio schedules of 10 and 20 does not change from a value of 25. However, it does change from a schedule of FR 20 to FR 30, with no signs of strain being evident on a schedule of FR 30. This animal, however, did show signs of strain on FR 60, and its distribution on this schedule has flattened with the mode lying somewhere between 45 and 105 laps per dipper presentation. All animals, when subjected to FR schedules of $10,20,30$, and 60 , tended to overlap at the dipper approximately $90 \%$ of the time.

The standard deviations of the lapping distributions for Ss 1, 2, 4, and 6 on ratio schedules of $10,20,30$, and 60 have indicated that as the schedule increased so also did the variability in the number of laps made at each dipper presentation. This marked increase in variability for Ss 1 and 6 can be seen in Fig. 2. The standard deviations for the lapping distributions for S-1 were 10.9 at FR 10, 6.0 at FR 20, and 8.1 at FR 30 . The standard deviation jumped to 29.6 at FR 60 where this S demon- strated ratio strain. For S-6, standard deviation values were 9.5 at FR $10,8.9$ at FR 20, and jumped to 21.1 at FR 30 where this S's first indication of strain was noted. Ss 2 and 4 showed the same increase in variability at the higher ratio values, but the increase was more gradual than in the Ss which did show strain. For example, S-4 had a standard deviation of 8.0 at FR 30 and moved up to 12.6 at FR 60 .

These data indicate that even though the dipper behavior was not subjected to any ratio reinforcement schedule, this member of the response chain was altered by the schedule that the animal was subjected to on the bar. Not only did the behavior change, but it appeared to change in a systematic fashion suggesting that certain variables, one of which is the schedule, influences other responses in the chain not directly related to bar pressing, the response which is under the control of the schedule.

\section{Referenees}

STELLAR, E., \& HILL, H. The rat's rate of drinking as a function of water deprivation. J. comp. physiol. Psychol., 1952, 45, 96-102.

WEISSMAN, A. Licking behavior of rats on a schedule of food reinforcement. Science, 1962, 135, 99-101.

\section{Notes}

1. This work was partially supported by Research Project No. 17-155 from the Illinois Psychiatric Training and Research Authority under the direction of the principle investigator, and partially by Research Grant: Clinical Psychology Research-Equipment, from the same agency under the direction of Professor Noble H. Kelley.

2. This work was conducted while the second author was under a USPHS traineeship- No. T1MH7817. 